\title{
La comunicazione come strumento infermieristico per l'empowerment del paziente in dialisi
}

Giornale di Tecniche Nefrologiche e Dialitiche 2019, Vol. 3I (I) 49-53

(C) The Author(s) 2019

Article reuse guidelines:

sagepub.com/journals-permissions DOI: I0.1 I77/0394936219837490

journals.sagepub.com/home/gtn

(3SAGE

\author{
Patrizia Quaranta', Debora Giudice'2, \\ Michele Fersurella', Giorgio Merola², \\ Graziano Minonne ${ }^{2}$ e Vincenzo Montinaro ${ }^{3}$
}

\begin{abstract}
Communication as an instrument for the empowerment of hemodialysis patients

When a patient with chronic renal failure is told he must undergo hemodialysis, he/she lives an emotional shock, followed by a phase of anxiety. If this shock can't be overcome, the patient can face some anxious-depressive pathologies that will complicate the already complex clinical picture.

The aim of this work is to demonstrate how the communicative/educational measures are the clinical instruments, applied especially by nurses, that lead the patient to the acquisition of different skills (such as self-reliance and psychosocial abilities).

In conclusion, an efficient communication, mainly based on active and participative listening, represents the most effective instrument for the patient with chronic-degenerative pathologies to promote the awareness of his own capacities of self-management, and to make him play a leading role in his own health.
\end{abstract}

\section{Keywords}

Hemodialysis patients, Educational interventions, Empowerment, Nursing

"Sentire non è altro che una facoltà permessa da uno dei nostri cinque sensi ma ascoltare è un'arte."

Frank Tyger

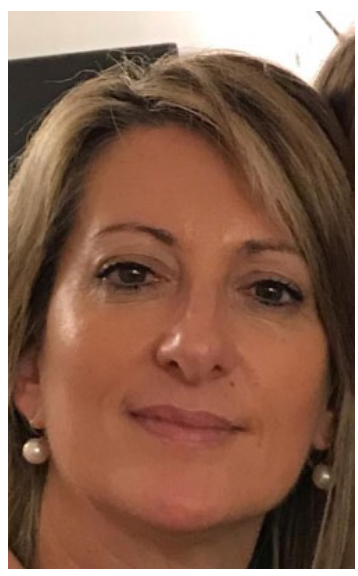

\section{Introduzione}

Nella malattia cronica il raggiungimento degli obiettivi terapeutici passa attraverso il paziente, per questo una buona comunicazione, quale elemento imprescindibile dell'educazione terapeutica, è indispensabile per prendersi cura in maniera efficace ed olistica della persona e della sua famiglia. I pazienti con insufficienza renale all'ultimo stadio (end-stage kidney disease, ESKD) sono esposti a fattori di rischio come il diabete, l'ipertensione, le alterazioni del metabolismo del calcio e del fosforo. Per modificare questi fattori il numero medio di farmaci da assumere varia da 6 a 12 compresse al giorno. ${ }^{1,2}$ La necessità di sottoporsi ai trattamenti depurativi e la forzata restrizione dell'apporto di liquidi costituisce un ulteriore elemento che condiziona la qualità della vita. ${ }^{3} \mathrm{La}$ mancata adesione allo schema dialitico, a causa degli eccessivi incrementi ponderali interdialitici (Interdialytic

\footnotetext{
IInfermiera Responsabile Servizio Prericoveri Ospedale "Card. G. Panico", Tricase (LE), Italy

2Infermiere U.O. Nefrologia e Dialisi Ospedale "Card. G. Panico",

Tricase (LE), Italy

3Medico Responsabile U.O.C. Nefrologia e Dialisi Ospedale "Card. G. Panico", Tricase (LE), Italy

Corrispondenza:

Dr.ssa Patrizia Quaranta, Infermiera Responsabile Servizio Prericoveri, Ospedale "Card. G. Panico", Via S. Pio X 4, 73039 Tricase (LE), Italy.

E-mail: patriziaq66@gmail.com
} 
Weight Gain, IDWG), può causare crampi muscolari, dispnea, edema polmonare e cardiomiopatie.., 5 In più l'IDWG costringe spesso, durante il trattamento dialitico, ad una deplezione rapida dei liquidi (ultrafiltrazione) che, con un inadeguato riempimento vascolare, unitamente ad alterazioni delle risposte vasoattive o simpatiche e della riserva cardiaca, svolge un ruolo fondamentale nella genesi dell'ipotensione indotta dalla dialisi (intra-dialytic hypotension, IDH). L'IDH determina una riduzione rilevante dell'efficienza dialitica, aumenta il rischio cardiovascolare ed è associata a una sintomatologia aspecifica (crampi agli arti inferiori o addominali, nausea, vomito, vertigini, sbadigliamento, ecc.). ${ }^{6}$ Recentemente, l'elenco dei problemi causati da IDH si è ulteriormente allungato per l'aggiunta della trombosi dell'accesso vascolare. ${ }^{7}$ I risultati degli studi sulle malattie renali, la dialisi e i trapianti confermano una bassa adesione a terapia e dieta, ${ }^{8,9}$ con una percentuale che varia dal $9.8 \%$ al $34.5 \%, 4,10$ con uno $0-7.9 \%$ di sedute disattese e un 7\%-32\% di riduzione della durata della seduta dialitica. ${ }^{3,7}$ Se le persone in cura non assumono i farmaci e non si attengono alla dieta, significa che fornire le migliori cure e la migliore assistenza tecnica non è sufficiente. A questo punto il problema diviene clinico: il personale sanitario ha l'esigenza di convincere il paziente a seguire le prescrizioni. Il solo modo per convincere qualcuno è ascoltare, parlare, negoziare, concordare.

\section{Comunicazione ed empowerment}

Ecco che la comunicazione diventa uno strumento dal quale non si può prescindere. ${ }^{11}$ Secondo il Settimo Rapporto del Joint National Committee on Prevention, Detection, Evaluation and Treatment of High Blood Pressure, ${ }^{12}$ "...la terapia più efficace prescritta dal più attento medico controllerà la malattia solo se i pazienti sono motivati." Nell'adottare l'empatia, i medici e il personale infermieristico svolgono un ruolo centrale. In particolare, gli infermieri di nefrologia e dialisi sono nella posizione ideale per stabilire una relazione con il paziente emodializzato cronico. ${ }^{13}$ Essi infatti, a livello mondiale, sono i professionisti sanitari più a contatto con i problemi quotidiani relativi alla dialisi (gestione del monitor, sicurezza, ipotensione, crampi, sanguinamento, trombosi dell'accesso vascolare, ecc.) e anche i primi ai quali i pazienti confidano le proprie difficoltà. Tuttavia, è importante che l'intervento dell'infermiere sia complementare a quello degli altri professionisti (medico, psicologo, dietista, assistente sociale, ecc.), perché il team multidisciplinare può promuovere l'acquisizione di diverse competenze (abilità di auto-cura e capacità psico-sociali). ${ }^{14}$

Nell'ambito della promozione della salute, si definisce empowerment il processo attraverso il quale le persone acquisiscono un maggiore controllo rispetto alle decisioni e alle azioni che riguardano la propria salute. ${ }^{15}$ Il tema dell'empowerment è stato affrontato in letteratura sulla base di analisi che hanno studiato il duplice ruolo di cittadino e operatore sanitario. Nel momento in cui il cittadino è anche operatore sanitario, è coinvolto in quanto risorsa di un'organizzazione sanitaria, ma anche come partner del cittadino, fruitore dei servizi. Questo duplice ruolo lo aiuta a perseguire gli obiettivi di salute, ricevendo nel contempo gratificazione e motivazione per la propria operatività, come professionista e come persona. ${ }^{16}$ La persona malata dovrebbe poter camminare con le proprie gambe ed autogestirsi, i curanti dovrebbero intervenire solo quando ci fosse mancata adesione alla terapia e alla dieta. Quando poi il paziente avesse comportamenti virtuosi non ci si dovrà attendere che questi siano stabili. ${ }^{11}$

\section{II processo psicologico del paziente in dialisi}

Quando ad un paziente con insufficienza renale cronica viene comunicato che dovrà sottoporsi ad emodialisi, il paziente vivrà un primo shock emotivo, seguito da una fase che ingenera ansia (l'approntamento della fistola artero-venosa, l'accesso alla procedura di emodialisi, il legare la propria vita in modo irreversibile ad una macchina, e ancora la perdita della funzione renale e della possibilità della minzione con la conseguente modificazione dell'immagine di sé stesso e del proprio schema corporeo). Se non si affronta tale shock, il paziente rischia l'insorgere di patologie ansioso-depressive, che complicano ancor più il già complesso quadro clinico. " $E$ in questo spazio operativo che si inserisce l'enorme lavoro di educazione terapeutica (ET) che è fatto di: informazione tecnica, supporto psicologico, interazione relazionale, personalizzazione del messaggio, concertazione delle possibilità e coinvolgimento del paziente. " 17 L'inizio della dialisi è, per il paziente, un cambiamento di tale entità da rappresentare praticamente una nuova diagnosi di malattia, che comporta, nella maggior parte dei casi, uno shock emotivo al quale segue un processo psicologico di cinque fasi (figura 1$){ }^{18}$

Il modello a cinque fasi secondo Kübler-Ross rappresenta uno strumento che permette di capire le dinamiche psicologiche più frequenti della persona a cui è stata diagnosticata una malattia grave. Da sottolineare che si tratta di un modello a fasi, e non a stadi, per cui le fasi possono anche alternarsi, presentarsi più volte nel corso del tempo, con diversa intensità, e senza un preciso ordine, dato che le emozioni non seguono regole, ma anzi come si manifestano, così svaniscono, magari miste e sovrapposte. Anche se la maggior parte delle persone sembra vivere le fasi secondo l'ordine in cui vengono qui descritte, non si tratta di un percorso "evolutivo a stadi", per cui le fasi possono manifestarsi in qualsiasi ordine e possono ripresentarsi successivamente, così come presentarsi sovrapposte. ${ }^{19}$ L'ascolto della diagnosi può produrre nel paziente un'anestesia emotiva che lo rende sordo alla 
Shock iniziale

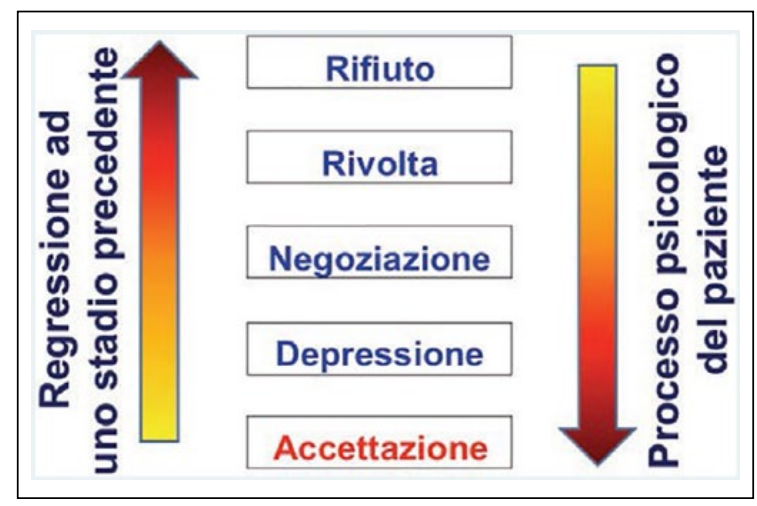

Figura I. II processo psicologico del paziente, a seguito di una malattia grave e/o cronica, dopo uno shock iniziale, consta di 5 differenti fasi. Tale processo giunto allo stadio di accettazione può regredire a stadi precedenti.

comunicazione. Al momento dello shock il medico deve essere conscio del fatto che nella maggior parte dei casi le sue parole andranno perse e il solo modo per dare istruzioni al paziente sarà farlo in forma scritta. Lo shock è seguito dalla fase detta di rifiuto, nella quale il paziente rifiuta di accettare ciò che gli è stato comunicato dal medico. Nega che la diagnosi lo riguardi: "non sono io", "non è vero", "si sono sbagliati" sono alcuni dei pensieri che possono descrivere questa fase. Segue una fase di rivolta nella quale il paziente pensa: "perché a me?". In questa fase la persona può essere colta da momenti di rabbia rivolta a cose o persone, compresi familiari e curanti. La fase detta di negoziazione vede il paziente, impaurito per il proprio futuro, negoziare con sé stesso: "se farò tutto ciò che i medici mi proporranno forse potrei guarire". Questo pensiero viene concepito a scapito delle affermazioni dei medici e al di là di ogni logica. In questa fase si possono alternare paura, speranza, rabbia e sconforto. La depressione è caratterizzata da tristezza e malinconia per le cose che si sono perdute e che mai potranno tornare. Il paziente si rende conto di non poter più fare cose che riteneva nella norma $\mathrm{e}$ che ora non farà più, in modo definitivo. L'accettazione è la fase della serenità, collaborazione, determinazione a fare qualcosa per sé stessi accettando la malattia e la terapia. Si sarebbe portati a pensare che quando il processo psicologico del paziente sia arrivato all'accettazione questa fase risulti stabile. Invece in moltissimi casi non è così! Il processo infatti è reversibile. Con i pazienti affetti da malattie croniche, il curante e il personale di assistenza devono essere consci del fatto che un paziente, che accetta la sua condizione di malattia da tempo, regredisca a stadi precedenti e che i suoi comportamenti, divenuti positivi, ritornino a essere negativi. In genere, per un paziente in fase di accettazione, un singolo evento negativo (riscontri analitici fuori norma, comparsa di una nuova sintomatologia, ma anche eventi personali stressanti) può causare il regresso ad uno stadio precedente del processo psicologico. I curanti devono aver presente che i pazienti andranno incontro a periodi positivi e negativi, più $o$ meno in modo alterno. Per tale motivo, comunicazione e relazione col paziente devono essere costanti e orientati a supportarlo al meglio. ${ }^{18}$

Per quanto concerne i soggetti dializzati, accanto alle fasi del processo psicologico del paziente, ci sono altre due fasi da considerare (tabella 1). ${ }^{20}$ La prima si verifica, di norma, quando il paziente sia sottoposto a dialisi da poco/ pochissimo tempo, ed è la fase detta della "luna di miele". In questo periodo i pazienti vivono bene la dialisi che dichiarano essere molto meno disagevole di quanto loro in precedenza descritto. Sono felici per il miglioramento delle personali condizioni di salute conseguenti alla dialisi. Le persone si sentono perfettamente in grado di far fronte alla malattia e di accettare i cambiamenti che questa comporta. I pazienti realizzano che gran parte dei loro timori erano infondati e si sentono in grado di poter convivere con la dialisi. In seguito, i dializzati possono entrare nella fase detta "di transizione" in cui vivono emozioni contrastanti, trascurano le raccomandazioni loro fatte e diffidano della parola dei sanitari. Spesso si sentono frustrati per il regime dietetico e terapeutico al quale vengono sottoposti. Possono avere sfiducia per le prospettive delle loro condizioni di salute o divenire euforici di fronte a una prospettiva di trapianto. Un qualsiasi cambiamento della terapia li mette in difficoltà e li spaventa. Alle due fasi descritte segue, normalmente, la fase di accettazione, nella quale il paziente dà la maggiore disponibilità ed è deciso ad operare positivamente per la sua salute. Il personale medico ed infermieristico dovrà tenere conto delle fasi psicologiche attraversate dal paziente, si relazionerà impiegando l'empatia, informando, educando alla terapia ed alla dieta. Il successo potrà essere rappresentato da una maggior fiducia del paziente nei confronti di medici e infermieri e da una maggior adesione alle prescrizioni. Educare le persone affette da malattie croniche può portare a risultati molto positivi, non è semplicissimo ma si può fare. L'importante è incominciare, almeno con quelle persone che sembrano essere più disponibili alla relazione. Sarà inevitabile commettere errori che potranno diminuire a mano a mano che si acquisisce esperienza. I primi successi ottenuti saranno di incoraggiamento: il successo genera altro successo e questo sarà la dimostrazione che la comunicazione può divenire uno strumento clinico.

\section{Storie ascoltate...}

"L'approccio con la dialisi è stato tremendo: avevo solo 18 anni. Pensavo durasse solo un giorno...la si fa, si sta meglio e si torna a casa. Dapprima l'impianto di un CVC poi...la fistola (un raccordo creato chirurgicamente tra una vena e un'arteria che sarà poi bucato da due grossi aghi fistola che 
Tabella I. Fasi di adattamento alla malattia renale cronica e considerazioni educazionali.

\begin{tabular}{|c|c|c|c|c|}
\hline Stadio & Descrizione & Componente psicosociale & $\begin{array}{l}\text { Considerazioni per gli } \\
\text { educatori }\end{array}$ & Obiettivi formativi \\
\hline Crisi iniziale & $\begin{array}{l}\text { Il paziente realizza di avere } \\
\text { una malattia renale cronica. }\end{array}$ & $\begin{array}{l}\text { - Shock } \\
\text { - Diniego } \\
\text { - Ansia } \\
\text { - Rabbia } \\
\text { - Paura }\end{array}$ & $\begin{array}{l}\text { In questa fase può essere } \\
\text { difficile far memorizzare } \\
\text { informazioni al paziente. }\end{array}$ & $\begin{array}{l}\text { Ricondurre alla } \\
\text { normalità e valorizzare } \\
\text { le reazioni emotive. }\end{array}$ \\
\hline Luna di miele & $\begin{array}{l}\text { Il paziente inizia ad } \\
\text { accettare l'idea di avere } \\
\text { un'insufficienza renale } \\
\text { cronica. }\end{array}$ & $\begin{array}{l}\text { Convinzione che la dialisi sia } \\
\text { meglio di come gli era stato } \\
\text { prospettato. }\end{array}$ & $\begin{array}{l}\text { Il paziente può } \\
\text { convincersi di essere un } \\
\text { "caso unico" e trascurare } \\
\text { i consigli dei medici. }\end{array}$ & $\begin{array}{l}\text { Normalizzare le } \\
\text { reazioni emotive. } \\
\text { Mettere a fuoco i limiti } \\
\text { della dialisi. }\end{array}$ \\
\hline Transizione & $\begin{array}{l}\text { Il paziente rinegozia } \\
\text { l'immagine di sé per poter } \\
\text { incorporare la gravità della } \\
\text { propria malattia. }\end{array}$ & $\begin{array}{l}\text { Dolore per la perdita della } \\
\text { salute. Frustrazione per le } \\
\text { limitazioni che comporta la } \\
\text { dialisi. Rabbia. Senso di colpa. } \\
\text { Depressione/tristezza. }\end{array}$ & $\begin{array}{l}\text { Il paziente può avere } \\
\text { diffidenza nei confronti } \\
\text { del personale sanitario } \\
\text { a causa delle emozioni } \\
\text { contrastanti. }\end{array}$ & $\begin{array}{l}\text { Aiutare il paziente } \\
\text { ad identificare le } \\
\text { motivazioni personali } \\
\text { verso un certo obiettivo } \\
\text { (dialisi-trapianto). }\end{array}$ \\
\hline Accettazione & $\begin{array}{l}\text { Il paziente } \\
\text { - Accetta la sua } \\
\text { condizione } \\
\text { - Fa programmi per il } \\
\text { futuro } \\
\text { - Assume comportamenti } \\
\text { positivi }\end{array}$ & $\begin{array}{l}\text { Migliore immagine di sé. } \\
\text { Maggiore adesione alla } \\
\text { terapia. }\end{array}$ & $\begin{array}{l}\text { Il paziente può essere } \\
\text { soddisfatto del } \\
\text { trattamento dialitico. }\end{array}$ & $\begin{array}{l}\text { Approvare i } \\
\text { cambiamenti positivi } \\
\text { nel comportamento del } \\
\text { paziente. }\end{array}$ \\
\hline
\end{tabular}

permettono l'aspirazione e la restituzione del sangue, dopo essere stato depurato da un "rene artificiale"). Incontro man mano chi vive la mia stessa condizione e mi chiedo come si fa ad accettare di dipendere da una macchina: il "mostro" tanto temuto, diventato poi il mio migliore compagno di viaggio".

Salvatore C., anni 42

"Quando faccio la dialisi sto abbastanza bene, mentre il mio sangue gira nei tubi,

io leggo, ascolto la musica e scherzo con i miei compagni di stanza, con il personale...

Ormai sono la mia seconda famiglia, mi sento a casa mia..."

Antonietta P., anni 33

"Siete i miei angeli, mi aiutate non solo quando mi sento male fisicamente, mi sostenete anche quando sono triste...non so come farei senza di voi".

Luisa B., anni 94

"La prima volta, quando ho capito che avrei dovuto fare la dialisi, è stato emotivamente massacrante. Mi chiedevo: perché proprio a me? Trovavo coraggio nella speranza di un trapianto. La seconda volta, dopo il rigetto e il ritorno in dialisi, mi sono preoccupato per mia moglie e per la mia nuova famiglia. Ora la vivo più serenamente, tra alti e bassi, ma sempre nella speranza di un altro trapianto. Ora che aspettiamo un figlio lo desidero più che mai... tutto è passato in secondo piano".

Rocco Roberto D. I., anni 51

\section{Conclusioni}

Gli strumenti più efficaci per promuovere nel paziente con patologie cronico-degenerative la consapevolezza delle proprie capacità di autogestione della patologia e renderlo protagonista della sua salute, come fruitore dei servizi ma anche come operatore sanitario, sono anzitutto l'informazione e la formazione attraverso una comunicazione efficace fatta, soprattutto, di ascolto attivo. Un ascolto attivo e partecipativo del paziente e dei familiari rappresenta un approccio olistico alla malattia perché dà la possibilità di raccontarsi ritrovando così le motivazioni perdute. Inoltre, svolge un ruolo importante anche il coinvolgimento di diverse figure di professionisti in un approccio multidisciplinare: nefrologo, infermiere, psicologo, dietista, fisioterapista, esperti in politiche sanitarie, esperti di bioetica. Naturalmente non esistono pazienti ideali, esistono solo pazienti con un vissuto personale e una storia clinica in continua evoluzione, dei quali il medico e il personale di assistenza tutto, pur non avendo una preparazione da psicologo, devono tener conto. Sarà necessario quindi conoscere la persona che seguiremo nel percorso dialitico, personalizzare la comunicazione con il paziente e i familiari, sia per stabilire la migliore dialisi in funzione della migliore qualità di vita del paziente, che per gestire la relazione con un malato difficile e la sua famiglia che chiederà molto all'operatore sul piano umano, professionale e personale. Una strategia fondata sull'idea di una dialisi in funzione della vita e non di una vita in funzione della dialisi. 


\section{Dichiarazione di assenza di conflitto di interessi}

Gli Autori dichiarano di non avere conflitti di interessi.

\section{Finanziamenti}

Gli Autori dichiarano di non aver ricevuto finanziamenti specifici da qualsiasi ente nei settori pubblico, privato o senza fini di lucro.

\section{Bibliografia}

1. Manley HJ, Cannella CA, Bailie GR and St Peter WL. Medication-related problems in ambulatory hemodialysis patients: a pooled analysis. Am J Kidney Dis 2005; 46: 669-680.

2. Kammerer J, Garry G, Hartigan M, Carter B and Erlich L. Adherence in patients on dialysis: strategies for success. Nephrol Nurs J 2007; 34:479-486.

3. Kluger C, Vlaminck H, Haverich A and Maes B. Nonadherence with diet and fluid restrictions among adults having hemodialysis. J Nurs Scholarsh 2005; 37(1):25-9.

4. Saran R, Bragg-Gresham J L, Rayner H C, et al. Nonadherence in hemodialysis: association with mortality, hospitalization, and practice patterns in the DOPPS. Kidney Int 2003; 64(1):254-62.

5. Goodman W G. Vascular calcification in end-stage renal disease. J Nephrol 2002; 15(Suppl. 6):S82-5.

6. Marchi G. Ipotensione intradialitica? Controlliamo anche la fistola! G Ital Nefrol 2011; 28(6):579.

7. Chang TI, Paik J, Greene T, et al. Intradialytic hypotension and vascular access thrombosis. J Am Soc Nephrol 2011; 22:1526-33.

8. Denhaerynck K, Manhaeve D, Dobbels F, et al. Prevalence and consequence of nonadherence to hemodialysis regimens. Am J Crit Care 2007; 16(3):222-235.

9. Carter S, Taylor D and Levenson R. A question of choice: compliance in medicine taking. University of London School of Pharmacy 2003.
10. Hecking E, Bragg-Gresham JL, Rayner $\mathrm{HC}$, et al. Haemodialysis prescription, adherence and nutritional indicators in five European countries: results from the Dialysis Outcome and Practice Patterns Study (DOPPS). Nephrol Dial Transplant 2004; 19(1):100-7.

11. Bertone B. "Abilità di comunicazione col paziente? Anche se fosse utile non saprei come cominciare!" TN\&D-Wichtig Editore 2011.

12. Chobanian AV, Bakris GL, Black HR, et al. The Seventh Report of the Joint National Committee on Prevention, Detection, Evaluation, and Treatment of High Blood Pressure (JNC 7). Hypertension 2003; 42:1206-1252.

13. Barnett T, Li Yoong T, Pinikahana J and Si-Yen T. Fluid compliance among patients having haemodialysis: can an educational programme make a difference? J Adv Nurs 2008; 61(3):300-6.

14. Idier L, Untas A, Koleck M, et al. Assessment and effects of Therapeutic Patient Education for patients in hemodialysis: A systematic review. International Journal of Nursing Studies 2011; 48:1570-1586.

15. World Health Organization Health Promotion Glossary. Ginevra 1998; 6.

16. Thema La centralità del paziente nell'organizzazione sanitaria. Supplemento a politiche sanitarie, 2011; 1:4.

17. Pegoraro M. Le alterazioni del metabolismo calcio-fosforo nei pazienti in dialisi: il contributo dello Studio DOPPS. Il punto di vista infermieristico. TN\&D-Wichtig Editore 2009.

18. Kubler-Ross E. Five stages of grief in On Death and Dying, Macmillan Publishing Co, NY, 1970.

19. Vivere il morire - le cinque fasi secondo Kubler Ross, 2008. available at: www.exit-italia.it/pdf/2008_01_KUBLER_ROSS CINQUE_FASI_DEL_MORIRE.pdf (ultimo accesso 1 novembre 2018).

20. Curtis CE, Rothstein $M$ and Hong BA. Stage-specific educational interventions for patients with end-stage renal disease: psychological and psychiatric considerations. 2009; 19(1):18-24. 